\title{
EVALUACIÓN DEL USO DE CORRIENTE ALTERNA EN LA MEDIDA DE LA RESPUESTA GALVÁNICA DE LA PIEL (GSR)
}

F.J. Verdú-García, J.M. Catalán, D. Martínez-Pascual, A. Blanco, J. Álvarez-Pastor, Nicolás García-Aracil Universidad Miguel Hernández de Elche, Instituto de Bioingenieria, francisco.verdug@umh.es

\section{Resumen}

Las terapias de rehabilitación asistidas por robot consiguen aumentar la motivación del paciente y la intensidad del ejercicio. Para ello, uno de los métodos con resultados muy prometedores es el uso de algoritmos de adaptación de la dificultad. Estos algoritmos se basan normalmente en parámetros de rendimiento del juego. Sin embargo, considerar el estado anímico del paciente podría mejorar la toma de decisiones en este tipo de estrategias. Una de las señales fisiológicas más utilizadas para la estimación del estado del paciente es el GSR. Los sensores de GSR utilizan corriente continua para realizar la medida de la conductancia de la piel. En este artículo, se propone el uso de corriente alterna a la hora de medir la conductancia de la piel. Este tipo de corriente puede aportar más información puesto que es capaz de penetrar a mayor profundidad en la piel del usuario. En este estudio se ha diseñado y evaluado un sensor de GSR de corriente continua y alterna para la estimación del estado del paciente durante un juego de rehabilitación con diferentes niveles de dificultad.

Palabras clave: Robótica de rehabilitación, respuesta fisiológica, GSR

\section{INTRODUCCIÓN}

La medida de la Respuesta Galvánica de la Piel (GSR) tiene aplicaciones en muchos ámbitos. En terapias de neurorehabilitación asistidas por robots, la señal GSR es una de las señales fisiológicas más utilizadas para medir diferencias en el estado anímico del paciente [4].

Tradicionalmente, la medida del GSR se ha llevado a cabo sometiendo la piel del usuario a una corriente o tensión continua a través de dos electrodos [1]. Si bien algunos autores [7] ya realizaron estudios sobre el uso corriente alterna para la medida del GSR, en algunos estudios se comenta la necesidad de realizar más estudios para analizar las ventajas que ofrece este tipo de técnica [2].
El método más común es medir la conductividad de la piel a través de una Corriente Continua (DC). Este método se ha mantenido durante años, sin embargo, las tecnologías de estos sensores basados en corriente continua han evolucionado con el tiempo [2].

Por su parte, emplear corriente Corriente Alterna (AC) para medir la GSR es un método poco convencional. A pesar de ello, este método muestra una serie de ventajas que potencialmente pueden resultar muy útiles a la hora de estudiar las diferencias en la respuestas fisiológicas de los usuarios. En primer lugar, en algunos estudios establecen que el modelo eléctrico de la piel no es meramente resistivo, sino que incluye componentes capacitivos [2]. El componente resistivo viene dado por la humedad presente en las capas internas de la piel, en concreto la dermis y la hipodermis, que las dota de conductividad, así como la presencia de las glándulas sudoríparas y su actividad en estas capas aumentan este efecto. Por su parte, el componente capacitivo viene dado por las diferencias de potencial que se generan entre las membranas de las glándulas sudoríparas y las células que las rodean. Las partes del cuerpo con mayor actividad glandular y, por tanto, con niveles mayores de GSR son las palmas de las manos y de los pies, mientras que zonas como la muñeca presentan menor actividad y las medidas tienen menos calidad [6].

En la Figura 1 se muestra el modelo eléctrico de la piel humana. Tal y como puede observarse, el condensador $C_{z}$ afecta de forma directa a la resistencia equivalente total, ya que aumentando la frecuencia de la señal que circula por el circuito, la impedancia $Z$ se reduciría debido al efecto del condensador, permitiendo medir con mayor facilidad en zonas donde el nivel medio de la señal GSR no sea muy alto.

Hay estudios que indican que el uso de corriente $\mathrm{AC}$ entre los electrodos reduciría el efecto de la polarización de los mismos que se da cuando se usa corriente DC [5]. Esta polarización genera una tensión de offset que es imposible de estimar, por lo que no se puede realizar una corrección a posteriori precisa. La corriente alterna, no 


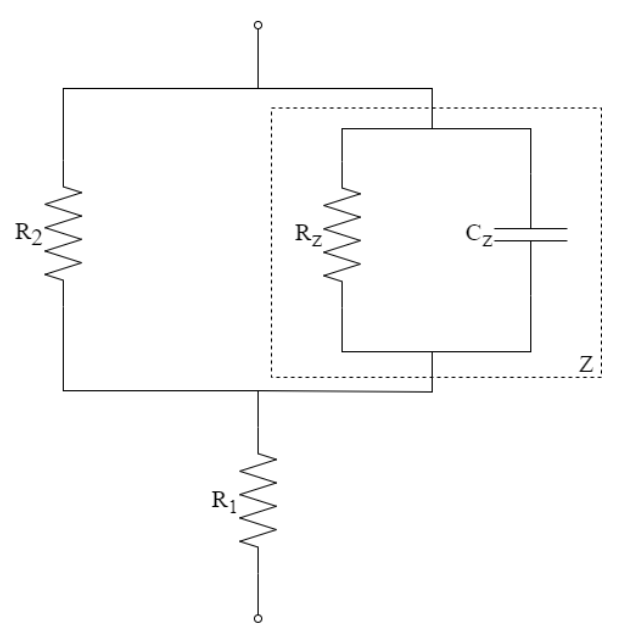

Figura 1: Modelo eléctrico de la piel humana. $R_{1}$ es la resistencia asociada a la dermis, mientras que $R_{2}$ es la resistencia de la epidermis. Ambas son valores constantes. $Z$ representa la impedancia variable por la acción de las glándulas sudoríparas. $[2]$

obstante, hace que el efecto de la polarización no sea tan grande, ya que las capacitancias formadas por la superficie de los electrodos y la piel se van cargando y descargando [3].

En este estudio se pretende evaluar el uso de la AC para la medida de la señal GSR durante un ejercicio de rehabilitación. Para ello, se analizarán las diferencias en las reacciones fisiológicas de diferentes usuarios realizando un ejercicio de rehabilitación con diferentes niveles de dificultad. Este método de medida se comparará con el método convencional basado en corriente DC.

\section{MATERIALES Y MÉTODOS}

\section{$2.1 \quad$ SUJETOS}

Han participado un total de 5 usuarios sin ningún tipo de discapacidad motora ni cognitiva (25, $40 \pm 2,06$ años). Todos ellos eran diestros. Fueron reclutados entre el personal del Dpto. de Ingeniería de Sistemas y Automática de la Universidad Miguel Hernández. La familiarización con robots de rehabilitación o juegos de ordenador no se consideró para su exclusión o inclusión en el estudio.

\subsection{MONTAJE EXPERIMENTAL}

La experimentación se ha llevado a cabo con el sujeto sentado frente a una mesa con ambos brazos reposando sobre ella. Los electrodos se han colocado en el brazo que el usuario no utilizará para realizar las pruebas. El sistema de medición se sitúa junto al brazo, a una distancia que impida que el circuito pueda ser golpeado por algún movimiento durante el ejercicio. En la mesa, frente al sujeto, se encuentra la pantalla en la que se muestra el juego, y el teclado con el que se realizaran las pruebas.

\subsection{MEDICIÓN DE LAS RESPUESTAS FISIOLÓGICAS}

\subsubsection{Diseño e Implementación del Sensor GSR}

Para llevar a cabo el experimento es necesario contar con un dispositivo de medición de conductividad, que se ha diseñado para dicho fin. El circuito es capaz de medir la conductividad de la piel usando dos fuentes de alimentación distintas: una que introduce una señal de tensión AC y otra que inyecta una tensión DC. El sensor ha sido validado mediante la comparación de medidas simultáneas del circuito diseñado y un dispositivo comercial, el Shimmer 3 GSR+ Unit.

Además, se debe contar con cuatro electrodos adhesivos, ya que cada canal, el de AC y el de DC, requiere de dos electrodos para realizar la medida. El circuito, controlado por un microcontrolador Teensy, consta de un amplificador operacional y varias resistencias y condensadores, formando una configuración de amplificador no inversor con un filtro paso-bajo a la salida y un divisor de tensión a la entrada, en la que una de las resistencias es la formada por la resistencia de la piel $\left(R_{s k}\right)$. El circuito está duplicado, habiendo uno por cada canal de medición, tal y como se aprecia en la Figura 2.

Así, al circuito se le introduce una tensión de entrada, que será o bien alterna o bien continua.

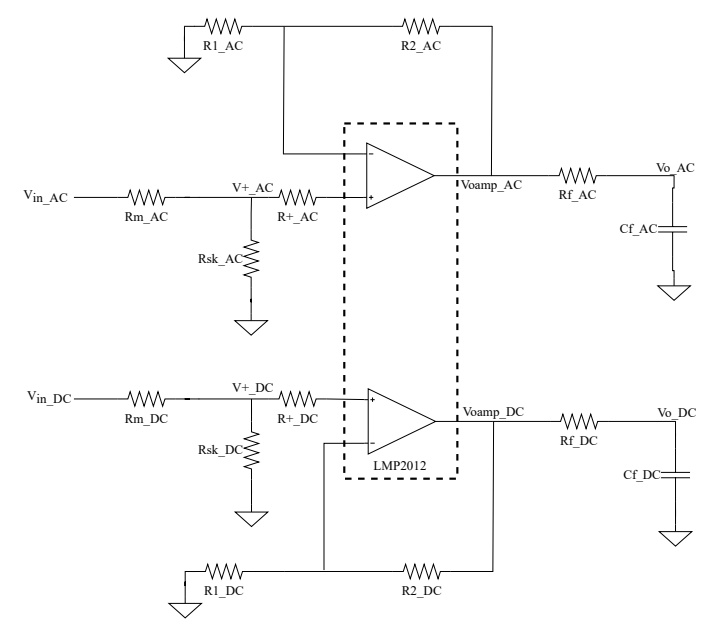

Figura 2: Esquemático del circuito con el que se ha realizado el experimento. 
Esta tensión variará en función de la resistencia de la piel tras su paso por el divisor de tensión y después se amplifica. Por último, la señal se filtra para que sea leída por el microcontrolador.

El circuito, pues, cuenta con dos canales de medida, uno para corriente continua y otro para corriente alterna.

\subsubsection{Medición mediante corriente continua}

La tensión de entrada en el canal DC es constante y tiene un valor de 3,3 voltios. Esta tensión se reduce tras el divisor de tensión, con un factor que depende de la resistencia de la piel. Posteriormente, esta tensión se introduce por la entrada positiva de un amplificador operacional, configurado como amplificador no inversor y con un factor de amplificación de 3. Finalmente, se filtra esta señal amplificada y se obtiene la tensión de salida del circuito, que es leída por el microcontrolador.

\subsubsection{Medición mediante corriente alterna}

El canal AC presenta un comportamiento funcional idéntico al de corriente continua, aunque existen algunas diferencias. La señal es, en este caso, un Pulso Modulado por Amplitud (PWM) de $1 \mathrm{MHz}$ de frecuencia y $50 \%$ de ciclo de trabajo y 3,3 voltios de amplitud. La señal es de frecuencia elevada para aprovechar los posibles beneficios que se le atribuyen [2].

La piel presenta unas propiedades eléctricas principalmente resistivas que tienen su origen en la humedad de las capas internas de la piel, así como en la segregación de sudor de las glándulas presentes en estas. No obstante, estas mismas glándulas son las que aportan a la impedancia de la piel su componente capacitiva, ya que entre sus membranas y el exterior se forman pequeñas diferencias de potencial que actúan como condensadores. Por ello es interesante el uso de señales AC, ya que la tensión continua ignora esos componentes capacitivos, mientras que la alterna interactúa con ellos.

Se espera, por tanto, que aún sin realizar un estudio frecuencial, pues es un análisis más complejo, el uso de tensión alterna ofrezca alguna mejora con respecto a la DC.

\subsection{PROTOCOLO}

Al inicio de la experimentación es importante informar al sujeto de en qué consiste la prueba que va a realizar, así como indicar que, durante las medidas, debe permanecer lo más inmóvil posible, en especial el brazo en el que están colocados los electrodos, para evitar la generación de artefactos en la señal medida. Una vez se han colocado los electrodos y se ha comprobado que están bien sujetos a la piel, se puede proceder al inicio de la prueba.

El presente estudio se ha llevado a cabo bajo dos condiciones, donde cada sujeto realizará la prueba con dos niveles de dificultad diferentes, con el fin de generar distintos niveles de respuestas fisiológicas en el usuario. De esta forma se pretende comprobar si, mediante las medidas de conductividad, existen diferencias entre los canales AC y DC para diversos estados del sujeto. Antes de cada prueba, se debe realizar una medida de baseline, durante la cual el sujeto debe estar lo más relajado posible, de forma que se logre una referencia de su estado de calma antes de estimular el estrés.

El orden de las pruebas, por su parte, es aleatorio para cada sujeto. La razón es que de esta manera se eliminan los factores debidos al orden de las pruebas y no condicionarán los resultados. Esto se ve en la Figura 3, en la que cada condición es un nivel de dificultad que se elige de forma aleatoria. Cada prueba, así como las baseline, tienen una duración de tres minutos y se realizarán dos veces, una por cada lugar de medida. Se realizan primero las medidas con los electrodos colocados en los dedos y, posteriormente, se repite el proceso con los electrodos en la muñeca. La gestión de la ejecución de los juegos y de la comunicación con el microcontrolador del circuito, así como del almacenamiento de las medidas, la realiza un programa central.

\section{$2.5 \quad$ Juego}

Para este estudio se ha utilizado el juego de rehabilitación, que se trata de un juego desarrollado específicamente para este tipo de pruebas en las que se pretende generar cierto estrés al usuario. El juego consiste en ganar

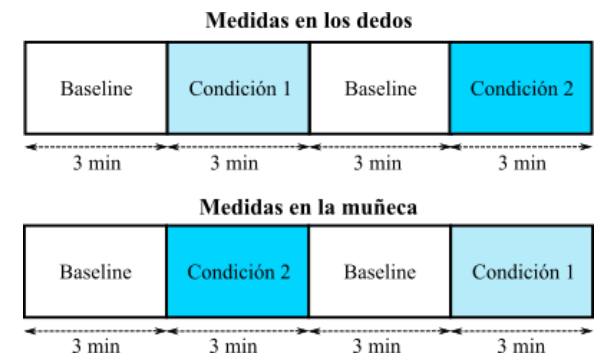

Figura 3: Diagrama de bloques del protocolo experimental. 
puntos evitando colisiones con los obstáculos, representados por rectángulos azules. Cada vez que se produce una colisión, se restan puntos. El jugador se representa por el cuadrado verde, que debe moverse hacia arriba, abajo, izquierda o derecha para evitar las colisiones, ya que los obstáculos tienen un movimiento errático y de carácter casi impredecible. El jugador puede moverse dentro de la zona de juego, que está delimitada por un recuadro.

Los niveles de dificultad se ajustan modificando el número de obstáculos, así como su velocidad de movimiento, de forma que el nivel fácil únicamente cuenta con un obstáculo y se mueve muy lento. Por su parte, el nivel difícil tiene tres obstáculos, cada uno con un movimiento distinto y, además, se mueven con mucha velocidad, por lo que predecir el movimiento de los tres para esquivarlos se vuelve muy complicado. El nivel difícil se puede observar en la Figura 4. Debido a estas configuraciones de dificultad, la mayoría de jugadores acaban con la puntuación perfecta en el nivel fácil, mientras que apenas son capaces de llegar, en general, a la mitad de la puntuación en el difícil.

\subsection{DATOS ADQUIRIDOS}

Durante el experimento, el sistema envía al programa central las medidas, tanto por el canal $\mathrm{AC}$ como por el canal DC, a una frecuencia de unas 16 muestras por segundo, frecuencia más que suficiente para medir el Nivel de Conductividad de la Piel (SCL) y la Respuesta de la Conductividad de la Piel (SCR), ya que la frecuencia de muestreo mínima necesaria es de $6 \mathrm{~Hz}[2]$. Cuando finaliza la toma de medidas, el programa almacena los dos grupos de datos junto con el tipo de prueba que se ha realizado. A la tensión medida se le aplica la conversión en conductancia según el circuito de la Figura 2 y un filtro de ventana para suavizar la señal.

Por tanto se obtienen dos grupos de medidas por cada prueba. Dado que cada sujeto realiza ocho,

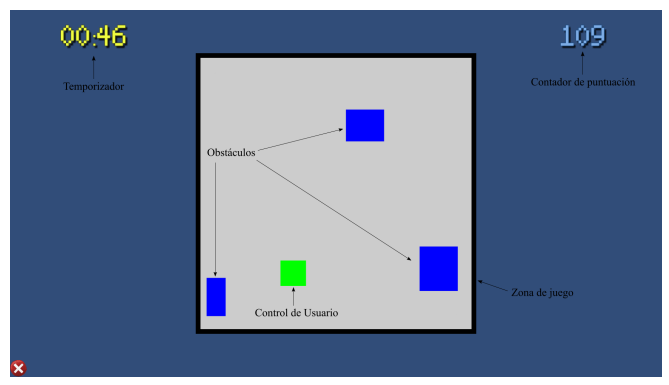

Figura 4: Captura de pantalla del juego de rehabilitación en nivel difícil. en total se tienen 16 grupos de medidas por sujeto, que posteriormente habrá que procesar y analizar.

El análisis se debe hacer de forma relativa a la baseline, debido a que el valor absoluto de conductividad puede variar mucho entre individuos y sería imposible realizar una comparación entre todas las medidas. Para ello, se van a procesar los datos de forma que se puedan comparar los SCLs de las distintas medidas.

Debido a que los valores de conductividad varían mucho de usuario a usuario, la comparación no puede realizarse directamente, sino que es necesario normalizar cada señal. Así, la normalización se hará usando como referencia la baseline. En primer lugar, se ha de calcular la media del rango final de la línea base $\left(\overline{B_{s}}\right)$ para establecer el nivel de reposo de cada individuo. Posteriormente, y usando esta media, se normaliza el valor de la conductancia respecto a este valor $\left(C_{n}\right)$. La fórmula se ve en la ecuación 1 . Se trata del cociente entre la resta del valor medio de la señal medida durante la prueba $(\bar{S})$ y el valor medio de la baseline y la resta del máximo de la señal entre la media de la línea de base. Con la resta de $\overline{B_{s}}$ se logra centrar todas las señales según cuánto se alejan de la media del estado de calma.

$$
C_{n}=\frac{\bar{S}-\overline{B_{s}}}{\max (\bar{S})-\overline{B_{s}}}
$$

\section{RESULTADOS}

El análisis de los resultados se ha realizado mediante su representación en un gráfico boxplot, en el que se representan los valores $C_{n}$ calculados para cada sujeto en cada una de las condiciones experimentales. En primer lugar, en la Figura 5 se pueden ver las medidas de las dos pruebas realizadas por los usuarios con los electrodos colocados en los dedos, es decir, el juego en nivel fácil y en nivel difícil. A la izquierda se muestran los valores correspondientes al canal AC, mientras que a la derecha se encuentran los del canal DC.

En la Tabla 1 se pueden ver los valores medios y la desviación típica de los coeficientes $C_{n}$ de los sujetos para cada condición.

Por su parte, en la gráfica de la Figura 6, está la representación de la conductividad normalizada para los mismos cuatro grupos de medidas tomadas en la muñeca: medidas AC para niveles fácil y difícil y medidas DC para niveles fácil y difícil. y en la Tabla 2 se exponen las medias y la desviación típica para cada grupo de medidas. 
Tabla 1: Media y desviación típica para las medidas realizadas en los dedos en alterna, continua y para las condiciones del juego en nivel fácil y difícil.

\begin{tabular}{|c|c|c|c|}
\hline Tensión & Dificultad & Media & D. Típica \\
\hline \multirow{2}{*}{ Alterna } & Fácil & 0.6249 & 0.2243 \\
\cline { 2 - 4 } & Difícil & 0.8307 & 0.0348 \\
\hline \multirow{2}{*}{ Continua } & Fácil & 0.6456 & 0.2223 \\
\cline { 2 - 4 } & Difícil & 0.8417 & 0.0327 \\
\hline
\end{tabular}

Tabla 2: Media y desviación típica para las medidas realizadas en la muñeca en alterna, continua y para las condiciones del juego en nivel fácil y difícil.

\begin{tabular}{|c|c|c|c|}
\hline Tensión & Dificultad & Media & D. Típica \\
\hline \multirow{2}{*}{ Alterna } & Fácil & -0.0714 & 0.4857 \\
\cline { 2 - 4 } & Difícil & 0.4138 & 0.5829 \\
\hline \multirow{2}{*}{ Continua } & Fácil & -0.1015 & 0.4991 \\
\cline { 2 - 4 } & Difícil & 0.3735 & 0.6162 \\
\hline
\end{tabular}

\section{DISCUSIÓN}

El principal objetivo que se planteó en este estudio es si mediante las medidas AC se podían diferenciar distintos niveles de GSR en comparación con las medidas en DC.

El sensor presentado se ha validado mediante un dispositivo comercial, el Shimmer 3 GSR+ Unit. Para la validación, se realizaron medidas simultáneas con ambos dispositivos, usando en el sensor presentado el canal DC. De esta forma, se puede comprobar si el sensor es capaz de detectar los cambios en las respuestas fisiológicas que el dispositivo comercial detecta, y tener así una estimación de la calidad de las medidas. No se realiza, sin embargo, calibración del valor exacto de conductividad medido, ya que, debido a la

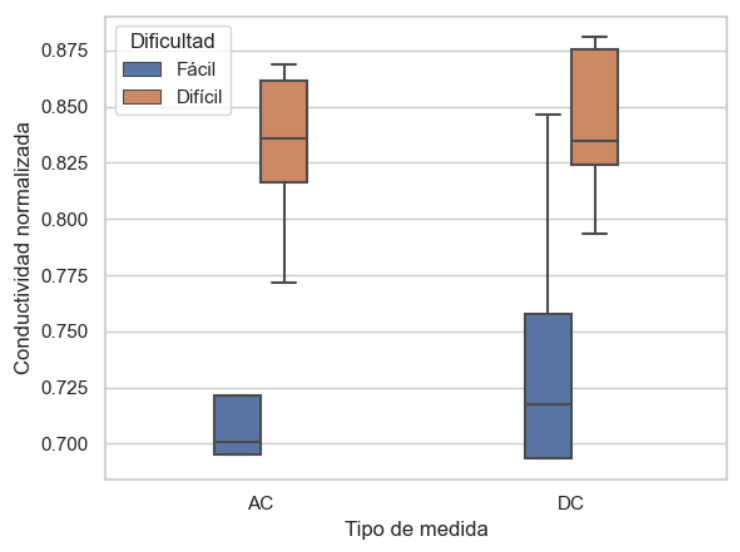

Figura 5: Gráfico boxplot de las medidas realizadas en los dedos.

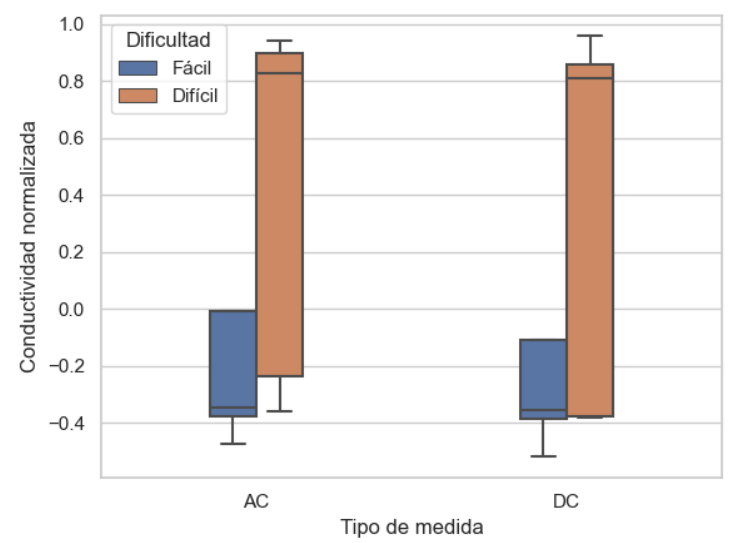

Figura 6: Gráfico boxplot de las medidas realizadas en la muñeca.

normalización, esta funcionalidad no es relevante. Así, en la Figura 7 se puede observar que ambos sensores realizan medidas muy similares, y que el sensor diseñado es capaz de seguir los aumentos y decrementos de la señal GSR.

En la gráfica de la Figura 5 se puede apreciar que para ambos canales parecen diferenciarse los dos niveles. La condición "fácil" presenta un nivel de GSR menor respecto a la baseline que la "difícil". Aunque para el canal DC el máximo del nivel fácil se solapa con el del nivel difícil, los recuadros no coinciden entre sí, por lo que se puede decir que ambos grupos de datos sí que se diferencian. Por otra parte, el rango de valores que presentan las medidas es relativamente pequeño, ya que todos se encuentran en 0.175 puntos. En la Tabla 1 se puede apreciar que ambos grupos de medidas, las AC y las DC, presentan propiedades muy parecidas, tanto en media como en desviación

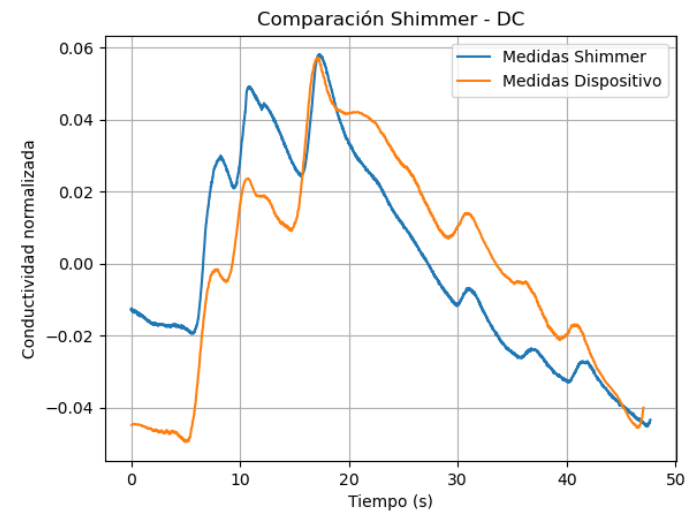

Figura 7: Validación entre la medida realizada con el sensor diseñado y la tomada con el Shimmer 3 GSR+ Unit. 
típica.

En la Figura 6 vemos la representación de las medidas en la muñeca. La calidad de estas medidas respecto a las realizadas en los dedos es mucho menor, lo que se evidencia porque los recuadros se solapan y es mucho más complicado diferenciar las dos condiciones, ya que los valores del nivel fácil están contenidos en los del difícil.

Además, vemos que en las medidas hay valores negativos, lo que quiere decir que las medidas tomadas durante las pruebas tenían un nivel de GSR menor al medido durante la baseline. Esto ocurre sobre todo en las medidas del nivel fácil, en las que los datos sugieren que los estímulos no son suficientes para generar una respuesta fisiológica medible, al menos en el nivel de la señal GSR.

En las medidas en nivel difícil también se pueden encontrar valores negativos, aunque para algunos sujetos la respuesta fisiológica producida sí que resultó medible para el sensor, lo que se traduce en un aumento considerable de la desviación típica, tal y como se observa en la Tabla 2 .

\section{CONCLUSIONES}

En este estudio se han comparado las medidas de GSR tomadas mediante corriente continua y mediante corriente alterna. Los resultados muestran que ambos métodos se comportan de forma muy similar. En el caso de las medidas en los dedos, tanto con AC como con DC parece que es posible distinguir ambos estados fisiológicos. En el caso de la muñeca, sin embargo, los datos muestran que la distinción es más complicada.

En resumen, se puede concluir que el uso de $\mathrm{AC}$ en la medida del GSR es, como mínimo, igual de válida que la tensión continua. Los datos apenas han mostrado diferencias entre ambos grupos, tanto en las medidas en los dedos como en las medidas en la muñeca. Esto es indicativo de que la medida en alterna tiene mucho potencial, ya que el funcionamiento de base es igual al de la medida en continua, por lo que con la implementación de un sistema capaz de analizar el espectro de la señal se podría obtener información sobre la parte imaginaria de la impedancia, cosa que es imposible mediante las medidas en DC. De esta forma se podría obtener información sobre la fase de la señal AC a la salida, o incluso realizar análisis espectrales que mostraran el efecto que tiene la piel sobre cada frecuencia.

\section{Agradecimientos}

Este estudio ha sido subvencionado por la Conselleria de Educación, Cultura y Deporte de la Generalitat Valenciana (GVA), a través de la ayuda ACIF/2018/214, de la ayuda APOTIP/2020/014 y de la ayuda APOTIP/2021/022 y por el Ministerio de Ciencia e Innovación, perteneciente a la Agencia Estatal de Investigación (AEI), a través del proyecto PID2019-108310RB-I00/AEI/10.13039/ 501100011033.

\section{Acrónimos}

GSR Respuesta Galvánica de la Piel

AC Corriente Alterna

DC Corriente Continua

SCL Nivel de Conductividad de la Piel

PWM Pulso Modulado por Amplitud

SCR Respuesta de la Conductividad de la Piel

\section{English summary}

EVALUATION OF ALTERNATING
CURRENT
MEASUREMENT OF GALVANIC
SKIN RESPONSE (GSR)

\section{Abstract}

Robot-assisted rehabilitation therapies get to improve pacient's motivation and exercise intensity. For this purpose, one of the methods with the most promising results is the use of difficulty-adapting algorithms. This algorithms are usually based in game performance parameteres. However, the consideration of the patient's emocional state could improve the decision making in this kind of strategies. One of the most used physiological signals for estimating the state of the patient is GSR. GSR sensors use direct current to measure skin conductance. In this article, the use of alternating current to measure skin conductance is proposed. This kind of current can provide more information because it can penetrate further in the user's skin. In this study, a GSR sensor capable of measuring both direct and alternating current was designed and evaluated in order to estimate the patient's 
state during a rehabilitation game with several difficulty levels.

Keywords: Rehabilitation robotics, physiologic response, GSR.

\section{Referencias}

[1] Badesa, F. J., Morales, R., Garcia-Aracil, N. M., Sabater, J. M., Zollo, L., Papaleo, E., Guglielmelli, E., (2016) Dynamic Adaptive System for Robot-Assisted Motion Rehabilitation, IEEE Systems Journal, vol. 10 , no. 3

[2] Boucsein, W., (2012) Electrodermal Activity, Springer

[3] Boucsein, W., Fowles, D. C., Grimmes, S., Ben-Shakhar, G., Roth, W. T., Dawson, M. E., Filion, D. L., (2012) Committee report: Publication recommendationsfor electrodermal measurements, Psychophysiology, vol 49.

[4] Catalán, J. M., García-Pérez, J. V., Blanco, A., Martínez, D., Lledó, L. D.,
García-Aracil, N., (2021) Differences in Physiological Reactions Due to a Competitive Rehabilitation Game Modality, sensors, vol. 21.

[5] Pabst, O., Tronstad, C., Grimnes, S., Fowles, D. C. and Martinsen, Ø. G., (2017) Comparison between the $\mathrm{AC}$ and DC measurement of electrodermal activity, Psychophysiology, vol. 54.

[6] van Dooren, M., de Vries, J. J. G., Janssen, J. H., (2012) Emotional sweating across the body: Comparing 16 different skin conductance measurement locations, Physiology \& Behavior, vol 106.

[7] Yamamoto, Y., Yamamoto, T., (1978) Dispersion and correlation of the parameters for skin impedance, Medical \& Biological Engineering \& Computing, vol. 16.

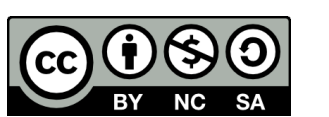

(C) 2021 by the authors. Submitted for possible open access publication under the terms and conditions of the Creative Commons Attribution CC BY-NC-SA 4.0 license (https://creativecommons.org/licenses/by-nc-sa/4.0/deed.es). 